\title{
Values of vector measure market games and their representations
}

\author{
Omer Edhan ${ }^{1}$
}

Accepted: 27 October 2015 / Published online: 23 November 2015

(C) The Author(s) 2015. This article is published with open access at Springerlink.com

\begin{abstract}
We offer a representation result for values of vector measure market games, proving that the value of a game is an "average of marginals". As a direct result we obtain that the Mertens value is the unique continuous value on the space of vector measure market games, and the unique value on the space of Lipschitz vector measure market games.
\end{abstract}

Keywords Shapley value $\cdot$ Nonatomic games $\cdot$ Market games

\section{Introduction}

The Shapley (1953) value is one of the basic solution concepts of cooperative game theory. Axiomatically, it was characterized by the linearity, efficiency, symmetry, and null player axioms. In various fields of economics and political science it is necessary to consider games that involve a large number of "insignificant" players. In such cases it is fruitful to model the game as a game with a continuum of players. Aumann and Shapley (1974) modified the value axioms and extended the definition of the value to games with a continuum of players. They proved the existence and uniqueness of the value on important spaces of nonatomic games. In computational terms, both the Shapley value and the Aumann-Shapley value assign each agent his average marginal

\footnotetext{
The main result and Theorem 3 in this work are based on ideas that first appeared in chapters 4 and 7 of the author's Ph.D. thesis done at the Hebrew University of Jerusalem under the supervision of Professor Abraham Neyman, whose advise, guidance and support are gratefully appreciated. This research was supported in part by the Israel Science Foundation Grants 1123/06 and 1596/10.
}

$凶$ Omer Edhan

omer.edhan@gmail.com

1 School of Social Sciences, The University of Manchester, Manchester M13 9PL, UK 
contribution to coalitions he may join, in certain models of random ordering of the agents.

On general classes of nonatomic games the value may not exist. Nevertheless, this is not the case when market games, derived from transferable utility (TU) perfectly competitive economies, are concerned. The core of a market game is nonempty, and if all agents have differentiable utility functions, then the Aumann-Shapley value is also well defined, and it constitutes the unique element of the core.

Without the differentiability assumption, the core of a market game is usually multivalued. But even in this scenario, all known values have the property of being elements of the core. This is the case with (Hart 1980) measure-based values of a nonatomic market game, and also with the (Mertens 1988) value (which is defined on a large space of games containing the linear space spanned by all nonatomic market games). In fact, in the special case of market games, the Mertens value is given by an explicit ${ }^{1}$ formula as a barycenter of the core (Mertens 1988).

The important and natural question that arises is whether the properties of the value can uniquely determine it, and make sure it is a member of the core. Arbitrary core selections can lead to distortions, e.g., significant differences in payoffs in "nearby" economies, discrimination between agents, and inconsistencies between choices. However, if the value indeed uniquely selects an element of the core, then the different choices of that element in different games are linked to each other via the value axioms, in a consistent and economically meaningful way.

This problem has proved to be extremely difficult. The first advancement in that direction was made by Haimanko (2002), essentially ${ }^{2}$ proving that if the games are functions of finitely many mutually singular probability measures ${ }^{3}$ then the standard value axioms together with the continuity axiom uniquely determine the Mertens value. Nevertheless, (Haimanko 2002) analysis heavily relies on the mutual-singularity assumption. It seems that a milestone in the way to characterize the value on the space of all market games would be to first eliminate the mutual singularity assumption.

One of the main tools developed by Haimanko (2002) is a representation of the value as an "average" of marginals of the underlying game. In this paper we prove a generalization of this result for the case of general vector measure market games. We then use this result to characterize the Mertens value as the unique continuous value on the space of vector measure market games. A subsequent result is a sufficient condition for the uniqueness of the value on other spaces of vector measure market games.

\section{Definitions, axioms, and the main result}

Let $(T, \mathcal{C})$ be a standard measure space. We shall call the members of $T$ players and the members of $\mathcal{C}$ coalitions. A game is a real valued function $v: \mathcal{C} \rightarrow \mathbb{R}$ s.t. $v(\emptyset)=0$.

\footnotetext{
1 A brief description of the formula is given in Appendix 2.

2 Haimanko characterization is expressed in terms of payoffs in economies. However, the result can be re-written in terms of values of market games.

3 Corresponding to economies of finite type, namely, with only finitely many types of utilities and endowments.
} 
A game $v$ is monotonic iff $v(S) \leq v\left(S^{\prime}\right)$ whenever $S \subseteq S^{\prime}$. If $Q$ is a set of games then $Q^{+}$denotes the subset of monotonic games in $Q$, and $Q^{1}$ denotes the subset $\left\{v \in Q^{+}: v(T)=1\right\}$. A game $v$ is of bounded variation iff it is the difference between two monotonic games. The space of all games of bounded variation will be denoted by $B V$. The variation of a game $v \in B V$ is the supremum of the variation of $v$ over all increasing chains $S_{0} \subseteq S_{1} \subseteq \ldots \subseteq S_{m}$ in $\mathcal{C}$, or equivalently,

$$
\|v\|=\inf \{u(T)+w(T): u, w \text { are monotonic games s.t. } v=u-w\} .
$$

The variation defines a norm on $B V$ (see Aumann and Shapley 1974). $F A$ denotes the subspace of $B V$ consisting of all finitely additive games, and $N A$ denotes its subspace consisting of nonatomic and countably additive measures.

Let $\Theta$ denote the group of measurable automorphisms ${ }^{4}$ of $(T, \mathcal{C})$. Each $\theta \in \Theta$ induces a linear mapping of $B V$ onto itself by ${ }^{5}(\theta v)(S)=v(\theta S)$. A linear subspace $Q \subseteq B V$ is symmetric iff $\theta Q=Q$ for each $\theta \in \Theta$.

Let $Q \subseteq B V$ be a symmetric space. A map $\varphi: Q \rightarrow B V$ is positive iff $\varphi\left(Q^{+}\right) \subseteq$ $B V^{+}$, symmetric iff $\theta \varphi=\varphi \theta$ for every $\theta \in \Theta$, and efficient iff $\varphi(v)(T)=v(T)$ for every $v \in Q$.

Definition 1 Let $Q \subseteq B V$ be a symmetric linear space. A value on $Q$ is a symmetric, positive and efficient linear map $\varphi: Q \longrightarrow F A$. A value is continuous iff it is continuous w.r.t. $\|\cdot\|_{B V}$.

A market function is a concave, continuous, nondecreasing, and homogeneous of degree 1 function on $\mathbb{R}_{+}^{k}$. Denote by $M_{+}^{k}$ the cone of market functions on $\mathbb{R}_{+}^{k}$, and let $M^{k}$ be the vector space of differences of functions in $M_{+}^{k}$. Denote by $L M_{+}^{k}$ the set of Lipschitz market functions, i.e., the subset of $M_{+}^{k}$ consisting of Lipschitz functions. Denote by $L M^{k}$ the vector space of differences of Lipschitz market functions. Denote by $\mathcal{M}$ the space of games of the form $f \circ \mu$ where $f \in M^{k}$ and $\mu \in\left(N A^{1}\right)^{k}$ for some $k \geq 2$. We refer to $\mathcal{M}$ as the space of vector measure market games. Denote by $\mathcal{L} \mathcal{M}$ the linear subspace of $\mathcal{M}$ consisting of games of the form $f \circ \mu$ where $f \in L M^{k}$ and $\mu \in\left(N A^{1}\right)^{k}$ for some $k \geq 2$.

In this work we shall prove a representation theorem for values on $\mathcal{L} \mathcal{M}$. This result will be formulated and developed in Sect. 3.3. It will be then employed to characterize the continuous values on $\mathcal{M}$ and the values on $\mathcal{L} \mathcal{M}$. It is known that the (Mertens 1988) value, $\Psi_{M}$, is continuous. Additionally, the Mertens value is entirely determined by the core of a market game, and is itself an element of the core (Mertens 1988). We shall prove that this selection is uniquely determined by the value and continuity axioms.

Theorem 1 The unique continuous value on $\mathcal{M}$ is the Mertens value.

Theorem 2 The unique value on $\mathcal{L} \mathcal{M}$ is the Mertens value.

\footnotetext{
4 Namely, bi-measurable bijections.

5 By abuse of notation.
} 


\section{Preparations}

\subsection{Directional derivatives of market functions}

Given $f \in M^{k}, x \in \mathbb{R}_{++}^{k}$, and $y \in \mathbb{R}^{k}$, the directional derivative $d f(x, y)$ of $f$ at $x$ in the direction $y$ is given by

$$
d f(x, y)=\lim _{\varepsilon \searrow 0} \frac{f(x+\varepsilon y)-f(x)}{\varepsilon} .
$$

The limit exists for every $f \in M_{+}^{k}$ by concavity.

If $f \in M_{+}^{k}$ then for every $x \in \mathbb{R}_{++}^{k}$ the function $d f(x, \cdot): \mathbb{R}^{k} \rightarrow \mathbb{R}$ is concave. Thus the directional derivative of $d f(x, \cdot)$ at $y \in \mathbb{R}^{k}$ in the direction $z \in \mathbb{R}^{k}$ which is given by

$$
d f(x, y, z)=\lim _{\varepsilon \searrow 0} \frac{d f(x, y+\varepsilon z)-d f(x, y)}{\varepsilon},
$$

exists. By linearity, Eqs. (2)-(3) extend to every $f \in M^{k}$.

Denote by $\mathbf{1}_{k} \in \mathbb{R}^{k}$ the vector with $\left(\mathbf{1}_{k}\right)_{1}=\ldots=\left(\mathbf{1}_{k}\right)_{k}=1$, and let $D^{k}=$ $\left\{t \mathbf{1}_{k}: t \in \mathbb{R}_{+}\right\}$. Notice that if $f \in M^{k}$ then for every $x \in D^{k} \backslash\left\{0_{k}\right\}$, every $y, z \in \mathbb{R}^{k}$, every $a \in \mathbb{R}$, and every $b>0$,

$$
d f\left(x, a \mathbf{1}_{k}+b y, z\right)=d f\left(\mathbf{1}_{k}, y, z\right) .
$$

For every $\chi: T \rightarrow \mathbb{R}^{k}, x \in \mathbb{R}_{++}^{k}$, and $y \in \mathbb{R}^{k}$ let $d f(x, y, \chi): T \rightarrow \mathbb{R}$ be given by

$$
\forall t \in T, d f(x, y, \chi)(t)=d f(x, y, \chi(t)) .
$$

\subsection{The direction space $X_{\lambda}$}

For every $k \geq 1$ and $\mu \in\left(N A^{1}\right)^{k}$ let $A F(\mu)$ be the vector space generated by the range of $\mu$, denoted $\mathcal{R}(\mu)$. Further denote ${ }^{6} \mathbb{S}_{\perp}^{\mu}=\left\{\frac{x}{\|x\|_{2}}: x \in A F(\mu), \bar{x}=0\right\}$, and $\Delta(\mu)=\left\{\frac{x}{k \bar{x}}: x \in \mathcal{R}(\mu) \backslash D^{k}\right\}$, with $\bar{x}=\frac{1}{k} \sum_{i=1}^{k} x_{i}$. For every $\mu \in\left(N A^{1}\right)^{k}$ endow the set $\Lambda_{\mu}=\Delta(\mu) \sqcup \mathbb{S}_{\perp}^{\mu}$ with the topology $\mathfrak{T}_{\mu}$ whose restriction to either $\Delta(\mu)$ or $\mathbb{S}_{\perp}^{\mu}$ is equivalent to the Euclidean topology, and if a sequence $\left(x^{n}\right)_{n=1}^{\infty} \subset \Delta(\mu)$ converges, in the Euclidean topology, to some point in $D^{k}$ and satisfies $\frac{x^{n}-\overline{x^{n}} \mathbf{1}_{k}}{\left\|x^{n}-\overline{x^{n}} \mathbf{1}_{k}\right\|_{2}} \underset{n \rightarrow \infty}{\longrightarrow} y \in \mathbb{S}_{\perp}^{\mu}$ then $x^{n} \underset{n \rightarrow \infty}{\longrightarrow} y$ in $\mathfrak{T}_{\mu}$. The topological space $\left(\Lambda_{\mu}, \mathfrak{T}_{\mu}\right)$ is thus a compact metrizable space.

For every $\lambda \in N A^{1}$ and $k \geq 1$ denote $\mathcal{Z}_{\lambda}^{k}=\left\{\mu \in\left(N A^{1}\right)^{k}: \bar{\mu} \ll \lambda, \frac{d \bar{\mu}}{d \lambda} \in L^{\infty}(\lambda)\right\}$, and $\mathcal{Z}_{\lambda}^{*}=\bigcup_{k=1}^{\infty} \mathcal{Z}_{\lambda}^{k}$. Let $B_{+}^{1}(T, \mathcal{C})$ be the set of bounded measurable functions $\chi$ :

6 The convention $\frac{0}{0}=0$ is used. 
$T \rightarrow \mathbb{R}$ with $0 \leq \chi \leq 1$. The direction space with perspective $\lambda$, denoted $X_{\lambda}$, is the closure of the image of $B_{+}^{1}(T, \mathcal{C})$ in $\prod_{\mu \in \mathcal{Z}_{\lambda}^{*}} \Lambda_{\mu}$, under the mapping

$$
y \mapsto(y(\mu))_{\mu \in \mathcal{Z}_{\lambda}^{*},}
$$

where for every $y \in B_{+}^{1}(T, \mathcal{C})$ and $\mu \in \mathcal{Z}_{\lambda}^{k} y(\mu)=\left\{\begin{array}{ll}\frac{\mu(y)}{k \overline{\mu(y)}}, & \mu(y) \notin D^{k} \\ 0_{k}, & \mu(y) \in D^{k}\end{array} . X_{\lambda}\right.$ is thus compact and Hausdorff, and every $x \in X_{\lambda}$ has the form $x=(x(\mu))_{\mu \in \mathcal{Z}_{\lambda}^{*}}$ with $x(\mu) \in \Lambda_{\mu}$ for every $\mu \in \mathcal{Z}_{\lambda}^{*}$.

\subsection{Representations of values on $\mathcal{L} \mathcal{M}$.}

Fix $\lambda \in N A^{1}$. For every $k \geq 2, f \in L M^{k}$ and $\mu \in \mathcal{Z}_{\lambda}^{k}$ the marginal $\partial(f, \mu): X_{\lambda} \rightarrow$ $L^{\infty}(\lambda)$ is given by

$$
\partial(f, \mu)(x)=\left\{\begin{array}{ll}
d f\left(x(\mu), \frac{d \mu}{d \lambda}\right), & x(\mu) \in \Delta(\mu) \\
d f\left(\mathbf{1}_{k}, x(\mu)+\mathbf{1}_{k}, \frac{d \mu}{d \lambda}\right), & x(\mu) \in \mathbb{S}_{\perp}^{\mu}
\end{array} .\right.
$$

Remark 1 Let $y \in B_{+}^{1}(T, \mathcal{C}), f \in L M^{k}$ and $\mu \in \mathcal{Z}_{\lambda}^{k}$, and suppose $\mu(y) \neq 0_{k}$. Then

$$
\partial(f, \mu)(y)=d f\left(\mu(y), \frac{d \mu}{d \lambda}\right)
$$

with $y \in X_{\lambda}$ on the left hand side of Eq. (8) and $y \in B_{+}^{1}(T, \mathcal{C})$ on the right hand side of the equation. Indeed, for $\mu(y) \notin D^{k}$ we have

$$
d f\left(\mu(y), \frac{d \mu}{d \lambda}\right)=d f\left(\frac{\mu(y)}{k \bar{\mu}(y)}, \frac{d \mu}{d \lambda}\right)=d f\left(y(\mu), \frac{d \mu}{d \lambda}\right)
$$

and for $\mu(y) \in D^{k} \backslash\left\{0_{k}\right\}$ we have

$$
d f\left(\mu(y), \frac{d \mu}{d \lambda}\right)=d f\left(\mathbf{1}_{k}, \mathbf{1}_{k}, \frac{d \mu}{d \lambda}\right)=d f\left(\mathbf{1}_{k}, y(\mu)+\mathbf{1}_{k}, \frac{d \mu}{d \lambda}\right)
$$

The following representation theorem will play an important role in the proof of our main result:

Theorem 3 Let $\varphi$ be a value on $\mathcal{L} \mathcal{M}$. For every $\lambda \in N A^{1}$ there is a finitely additive, positive vector measure $P_{\lambda}$ of bounded semi-variation (i.e., $\left|P_{\lambda}\right|\left(X_{\lambda}\right)<\infty$. See Appendix 3 for details.) on the Borel sets of $X_{\lambda}$ with values in $\mathcal{L}\left(L^{\infty}(\lambda), L^{2}(\lambda)\right)$ s.t. for every coalition $S \in \mathcal{C}$ the vector measure $P_{\lambda}^{S}=\left\langle P_{\lambda}, \chi_{S}\right\rangle$ is positive, regular, and 
countably additive of bounded variation, and for every $f \in L M^{k}$ and $\mu \in \mathcal{Z}_{\lambda}^{k}$ we have for every $S \in \mathcal{C}$

$$
\varphi(f \circ \mu)(S)=\int_{X_{\lambda}} \partial(f, \mu)(x) d P_{\lambda}^{S}(x)
$$

and in case that also ${ }^{7} \partial(f, \mu) \in C\left(X_{\lambda}, L^{\infty}(\lambda)\right)$ then

$$
\varphi(f \circ \mu)(S)=\int_{X_{\lambda}} \partial(f, \mu)(x) d P_{\lambda}^{S}(x)=\int_{S}\left(\int_{X_{\lambda}} \partial(f, \mu)(x) d P_{\lambda}(x)\right) d \lambda
$$

Theorem 3 is proved in Appendix 1.

Lemma 1 For every $\lambda \in N A^{1}$ and $\chi \in L^{\infty}(\lambda)$ the vector measure $P_{\lambda}$ in Theorem 3 satisfies

$$
\left\langle\chi, P_{\lambda}\right\rangle(E)=\chi\left\langle 1, P_{\lambda}\right\rangle(E)
$$

for every Borel set $E \subseteq X_{\lambda}$.

Lemma 1 is proved in Appendix 1.

\subsection{Values of exact market games}

For every $k \geq 2$ denote by $\Delta^{k}$ the $k-1$ dimensional simplex in $\mathbb{R}_{+}^{k}$. The space of exact market games, $\mathcal{E} \mathcal{M}$, is the linear space spanned by games of the form $v(S)=$ $\min _{c \in C} c \cdot \mu(S)$, for a compact and convex $C \subseteq \Delta^{k}$ and $\mu \in\left(N A^{1}\right)^{k}$, for some $k \geq 2$. The following Theorem was in fact proved in Edhan (2015):

Theorem 4 The Mertens value is the unique value on $\mathcal{E} \mathcal{M}$.

Remark 2 As $\mathcal{E} \mathcal{M} \subseteq \mathcal{M}$ the Mertens value is well defined on $\mathcal{E} \mathcal{M}$ by Eq. (61) (in the Appendix). Note that in Theorem 4 we do not require the continuity axiom.

\section{The proof}

Let $\varphi$ be a continuous value on $\mathcal{M}$. By (Edhan 2015, Corollary 1), $\varphi$ is uniquely determined by its values on $\mathcal{L} \mathcal{M}$. The restriction of $\varphi$ to $\mathcal{L} \mathcal{M}$ is a continuous value on $\mathcal{L} \mathcal{M}$. By abuse of notation we shall denote it by $\varphi$. Then:

Proposition $1 \varphi$ is the Mertens value.

To prove Proposition 1, which will in fact prove Theorems 1 and 2, we shall need the following Lemmata.

$\overline{7} C\left(X_{\lambda}, L^{\infty}(\lambda)\right)$ is the space of continuous functions from $X_{\lambda}$ to $L^{\infty}(\lambda)$ endowed with its uniform topology. 
Lemma 2 For every $w \in \mathbb{S}_{\perp}^{\mu} \backslash\left\{0_{k}\right\}$ and every sufficiently small $\epsilon>0$ there is a continuous and positively homogeneous of degree 1 function $h_{\epsilon}^{w}: \mathbb{R}_{+}^{k} \rightarrow \mathbb{R}$ which is twice continuously differentiable on $\mathbb{R}_{+}^{k} \backslash\left\{0_{k}\right\}$, vanishes on the conical diagonal neighborhood $N_{\epsilon}=\left\{x: \sum_{\ell=1}^{k}\left|x_{\ell}-\bar{x}\right|<\epsilon k \bar{x}\right\}$, and for every $z \in \mathbb{R}_{+}^{k}$ with $w \cdot z \neq 0$ and every $1 \leq \ell \leq k$ we have $\frac{\partial h_{\epsilon}^{w}}{\partial x_{\ell}}(z) \underset{\epsilon \rightarrow 0^{+}}{\longrightarrow} w_{\ell}$, where the convergence is uniformly bounded in $\epsilon$.

Proof Choose a continuously twice differentiable function $g_{\epsilon}: \mathbb{R} \rightarrow \mathbb{R}$ satisfying $g_{\epsilon}=0$ on $[-\epsilon, \epsilon], g_{\epsilon}^{\prime}=1$ on $[-2 \epsilon, 2 \epsilon]^{c}$, and $0 \leq g_{\epsilon}^{\prime} \leq 1$ on $\mathbb{R}$. Define $h_{\epsilon}^{w}: \mathbb{R}_{+}^{k} \rightarrow \mathbb{R}$ by

$$
h_{\epsilon}^{w}(x)= \begin{cases}k \bar{x} g_{\epsilon}\left(\frac{w \cdot x}{k \bar{x}}\right), & x \neq 0_{k} \\ 0, & x=0_{k}\end{cases}
$$

The function $h_{\epsilon}^{w}$ is continuous and positively homogeneous of degree 1 on $\mathbb{R}_{+}^{k}$, and it is twice continuously differentiable on $\mathbb{R}_{+}^{k} \backslash\left\{0_{k}\right\}$. It also vanishes on $N_{\epsilon}$; indeed if $x \in N_{\epsilon} \backslash\left\{0_{k}\right\}$ then

$$
\left|\frac{w \cdot x}{k \bar{x}}\right|=\left|w \cdot\left(\frac{x}{k \bar{x}}-\frac{1}{k} \mathbf{1}_{k}\right)\right| \leq \sum_{i=1}\left|\frac{x_{i}}{k \bar{x}}-\frac{1}{k}\right|<\epsilon,
$$

hence $h_{\epsilon}^{w}(x)=g_{\epsilon}\left(\frac{w \cdot x}{k \bar{x}}\right)=0$.

As to the convergence of the partial derivative for $z \in \mathbb{R}_{+}^{k}$ with $w \cdot z \neq 0$, notice that

$$
\frac{\partial h_{\epsilon}^{w}}{\partial x_{\ell}}(z)=g_{\epsilon}\left(\frac{w \cdot z}{k \bar{z}}\right)+\frac{k \bar{z} w_{\ell}-w \cdot z}{k \bar{z}} g_{\epsilon}^{\prime}\left(\frac{w \cdot z}{k \bar{z}}\right) \underset{\epsilon \rightarrow 0^{+}}{\longrightarrow} w_{\ell} .
$$

Also notice that the convergence is indeed uniformly bounded w.r.t. $\epsilon$.

Lemma 3 Let $\mu \in \mathcal{Z}_{\lambda}^{k}$ with $\operatorname{dim}(A F(\mu)) \geq 2$, and denote $S_{\mu}=\left\{s \in T: \frac{d \mu}{d \lambda}(s) \notin\right.$ $D^{k}$. Then (in the vector lattice $L^{2}(\lambda)$ )

$$
\left\langle\chi_{S_{\mu}}, P_{\lambda}\right\rangle\left(\left\{x \in X_{\lambda}: x(\mu) \notin \mathbb{S}_{\perp}^{\mu}\right\}=0 .\right.
$$

Proof There is a Borel set $\Omega \subset \mathbb{S}_{\perp}^{\mu}$ of Haar measure 1 s.t. for every $w \in \Omega$ we have $\left\langle 1, P_{\lambda}^{T}\right\rangle\left(\left\{x \in X_{\lambda}: x(\mu) \notin \mathbb{S}_{\perp}^{\mu}, w \cdot x(\mu)=0\right\}\right)=0$. Hence for every $S \in \mathcal{C}$ and every $w \in \Omega$ we have $\left\langle 1, P_{\lambda}^{S}\right\rangle\left(\left\{x \in X_{\lambda}: x(\mu) \notin \mathbb{S}_{\perp}^{\mu}, w \cdot x(\mu)=0\right\}\right)=0$. Choose $w \in \Omega$ and given $\epsilon>0$ let choose the function $h_{\epsilon}^{w}$ as in Lemma 2 . Then for any sufficiently small $\epsilon>0,\left\|\partial\left(h_{\epsilon}^{w}, \mu\right)\right\|_{\infty}$ is uniformly bounded w.r.t. $\epsilon$, and for every $x \in X_{\lambda}$ with $x(\mu) \notin \mathbb{S}_{\perp}^{\mu}$ and $w \cdot x(\mu) \neq 0$ we have $\partial\left(h_{\epsilon}^{w}, \mu\right)(x) \underset{\epsilon \rightarrow 0^{+}}{\longrightarrow} \frac{d(w \cdot \mu)}{d \lambda}$ in the $L^{\infty}(\lambda)$ norm. 
Thus

$$
\begin{aligned}
\varphi\left(h_{\epsilon}^{w} \circ \mu\right)(S)= & \int_{X_{\lambda}} \partial\left(h_{\epsilon}^{w}, \mu\right)(x) d P_{\lambda}^{S}(x) \\
= & \int_{\left\{x \in X_{\lambda}: x(\mu) \notin \mathbb{S}_{\perp}^{\mu}\right\}} d h_{\epsilon}^{w}\left(x(\mu), \frac{d \mu}{d \lambda}\right) d P_{\lambda}^{S}(x) \underset{\epsilon \rightarrow 0^{+}}{\longrightarrow} \\
& \left\langle\frac{d(w \cdot \mu)}{d \lambda}, P_{\lambda}^{S}\right\rangle\left(\left\{x \in X_{\lambda}: x(\mu) \notin \mathbb{S}_{\perp}^{\mu}\right\}\right),
\end{aligned}
$$

where the convergence in line (16) follows from the bounded convergence theorem 6 (in the Appendix). By (Edhan 2015, Theorem 1) we have $\varphi\left(h_{\epsilon}^{w} \circ \mu\right)(S)=0$, and by combining that with Eq. (16) we obtain for every $w \in \Omega$

$$
\begin{aligned}
\forall S \in \mathcal{C}, \quad 0 & =\left\langle\frac{d(w \cdot \mu)}{d \lambda}, P_{\lambda}^{S}\right\rangle\left(\left\{x \in X_{\lambda}: x(\mu) \notin \mathbb{S}_{\perp}^{\mu}\right\}\right) \Rightarrow \\
0 & =\left\langle\frac{d(w \cdot \mu)}{d \lambda}, P_{\lambda}\right\rangle\left(\left\{x \in X_{\lambda}: x(\mu) \notin \mathbb{S}_{\perp}^{\mu}\right\}\right),
\end{aligned}
$$

where the equality in line (18) holds in $L^{2}(\lambda)$ and follows by taking the RadonNikodym derivative in line (17). By combining Eq. (11) in Lemma 1 together with Eq. (18) we obtain for every $w \in \Omega$ and $\lambda$-a.e. $s \in T$ with $\frac{d(w \cdot \mu)}{d \lambda}(s) \neq 0$ that

$$
0=\left\langle 1, P_{\lambda}\right\rangle\left(\left\{x \in X_{\lambda}: x(\mu) \notin \mathbb{S}_{\perp}^{\mu}\right\}\right)(s) .
$$

As $\Omega$ is of full Lebesgue measure in $\mathbb{S}_{\perp}^{\mu}$ we thus conclude that

$$
0=\left\langle 1, P_{\lambda}\right\rangle\left(\left\{x \in X_{\lambda}: x(\mu) \notin \mathbb{S}_{\perp}^{\mu}\right\}\right)(s)
$$

for $\lambda$-a.e. $s \in T$ with $\frac{d \mu}{d \lambda}(s) \notin D^{k}$, hence, by Lemma 1 , we have (in the vector lattice $\left.L^{2}(\lambda)\right)$

$$
0=\left\langle\chi_{S_{\mu}}, P_{\lambda}\right\rangle\left(\left\{x \in X_{\lambda}: x(\mu) \notin \mathbb{S}_{\perp}^{\mu}\right\}\right)
$$

It is sufficient to verify Proposition 1 for games $f \circ \mu$ with $f \in L M_{+}^{k}$ and $\mu \in$ $\left(N A^{1}\right)^{k}$ with $\operatorname{dim}(A F(\mu)) \geq 2$. For such $f$ let $h(f): \mathbb{R}_{+}^{k} \rightarrow \mathbb{R}$ be given by $h(f)(x)=d f\left(\mathbf{1}_{k}, x\right)$. Then $h(f) \circ \mu \in \mathcal{E} \mathcal{M}$. Hence, for every $S \in \mathcal{C}$

$$
\begin{aligned}
\varphi(f \circ \mu)(S) & =\int_{X_{\bar{\mu}}} \partial(f, \mu)(x) d P_{\bar{\mu}}(x) \\
& =\int_{\left\{x \in X_{\bar{\mu}}: x(\mu) \notin \mathbb{S}_{\perp}^{\mu}\right\}} d f\left(x(\mu), \frac{d \mu}{d \bar{\mu}}\right) d P_{\bar{\mu}}(x)
\end{aligned}
$$




$$
\begin{aligned}
& +\int_{\left\{x \in X_{\bar{\mu}}: x(\mu) \in \mathbb{S}_{\perp}^{\mu}\right\}} d f\left(\mathbf{1}_{k}, x(\mu), \frac{d \mu}{d \bar{\mu}}\right) d P_{\bar{\mu}}(x) \\
& =\int_{\left\{x \in X_{\bar{\mu}}: x(\mu) \notin \mathbb{S}_{\perp}^{\mu}\right\}} d f\left(x(\mu), \frac{d \mu}{d \bar{\mu}} \chi S_{\mu}^{c}\right) d P_{\bar{\mu}}(x) \\
& +\int_{\left\{x \in X_{\bar{\mu}}: x(\mu) \notin \mathbb{S}_{\perp}^{\mu}\right\}} d f\left(x(\mu), \frac{d \mu}{d \bar{\mu}} \chi_{S_{\mu}}\right) d P_{\bar{\mu}}(x) \\
& +\int_{\left\{x \in X_{\bar{\mu}}: x(\mu) \in \mathbb{S}_{\perp}^{\mu}\right\}} d f\left(\mathbf{1}_{k}, x(\mu), \frac{d \mu}{d \bar{\mu}}\right) d P \frac{S}{\mu}(x) \\
& =\int_{\left\{x \in X_{\bar{\mu}}: x(\mu) \notin \mathbb{S}_{\perp}^{\mu}\right\}} d f\left(x(\mu), \chi_{S_{\mu}^{c}} \mathbf{1}_{k}\right) d P_{\bar{\mu}}^{S}(x) \\
& +\int_{\left\{x \in X_{\bar{\mu}}: x(\mu) \in \mathbb{S}_{\perp}^{\mu}\right\}} d h(f)\left(\mathbf{1}_{k}, x(\mu), \frac{d \mu}{d \bar{\mu}}\right) d P_{\bar{\mu}}(x) \\
& \geq \int_{\left\{x \in X_{\bar{\mu}}: x(\mu) \notin \mathbb{S}_{\perp}^{\mu}\right\}} d h(f)\left(x(\mu), \chi_{S_{\mu}^{c}} \mathbf{1}_{k}\right) d P_{\bar{\mu}}^{S}(x) \\
& +\int_{\left\{x \in X_{\bar{\mu}}: x(\mu) \in \mathbb{S}_{\perp}^{\mu}\right\}} d h(f)\left(\mathbf{1}_{k}, x(\mu), \frac{d \mu}{d \bar{\mu}}\right) d P_{\bar{\mu}}(x) \\
& =\int_{\left\{x \in X_{\bar{\mu}}: x(\mu) \notin \mathbb{S}_{\perp}^{\mu}\right\}} d h(f)\left(x(\mu), \frac{d \mu}{d \bar{\mu}} \chi_{S_{\mu}}\right) d P_{\bar{\mu}}(x) \\
& +\int_{\left\{x \in X_{\bar{\mu}}: x(\mu) \notin \mathbb{S}_{\perp}^{\mu}\right\}} d h(f)\left(x(\mu), \chi_{S_{\mu}^{c}} \mathbf{1}_{k}\right) d P \frac{S}{\mu}(x) \\
& +\int_{\left\{x \in X_{\bar{\mu}}: x(\mu) \in \mathbb{S}_{\perp}^{\mu}\right\}} d h(f)\left(\mathbf{1}_{k}, x(\mu), \frac{d \mu}{d \bar{\mu}}\right) d P_{\bar{\mu}}(x) \\
& =\int_{\left\{x \in X_{\bar{\mu}}: x(\mu) \notin \mathbb{S}_{\perp}^{\mu}\right\}} d h(f)\left(x(\mu), \frac{d \mu}{d \bar{\mu}}\right) d P_{\bar{\mu}}(x) \\
& +\int_{\left\{x \in X_{\bar{\mu}}: x(\mu) \in \mathbb{S}_{\perp}^{\mu}\right\}} d h(f)\left(\mathbf{1}_{k}, x(\mu), \frac{d \mu}{d \bar{\mu}}\right) d P_{\bar{\mu}}(x)
\end{aligned}
$$




$$
\begin{aligned}
& =\int_{X_{\bar{\mu}}} d h(f)\left(\mathbf{1}_{k}, x(\mu), \frac{d \mu}{d \bar{\mu}}\right) d P_{\bar{\mu}}(x)=\varphi(h(f) \circ \mu)(S) \\
& =\Psi_{M}(h(f) \circ \mu)(S)=\Psi_{M}(f \circ \mu)(S),
\end{aligned}
$$

where the first equality in line (22) follows from Theorem (3), the equality in line (23) follows by combining Lemma 3 with the definition of $h(f)$, the inequality in line (24) follows as $d f\left(z, \mathbf{1}_{k}\right) \geq f\left(\mathbf{1}_{k}\right)=h(f)\left(\mathbf{1}_{k}\right)=d h(f)\left(z, \mathbf{1}_{k}\right)$ for every $z \in \mathbb{R}_{+}^{k} \backslash D^{k}$, the equality in line (25) follows from Lemma 3 , the equality in line (26) follows as $d h(f)(z, x)+d h(f)\left(z, a \mathbf{1}_{k}\right)=d h(f)\left(z, x+a \mathbf{1}_{k}\right)$ for every $z \in \mathbb{R}_{+}^{k} \backslash D^{k}, x \in \mathbb{R}_{+}^{k}$, and $a \in \mathbb{R}_{+}$, the first equality in line (27) follows from Theorem 3 , and the second equality in line (27) follows from Theorem 4.

Now, by the efficiency axiom we obtain for every $S \in \mathcal{C}$

$$
\varphi(f \circ \mu)(S)=\Psi_{M}(f \circ \mu)(S),
$$

and we are done.

\section{An alternative axiomatization}

As mentioned in the introduction, the axioms of positivity and continuity [axioms (4) and (5) respectively] can be replaced by the axiom of contraction [axiom (4')]. Indeed, suppose that a map $\Psi: \mathcal{M} \rightarrow F A_{+}$obeys the axioms of efficiency and contraction. Then $\Psi$ is obviously continuous. Furthermore, by (Aumann and Shapley 1974, Proposition 4.6) $\Psi$ also obeys the positivity axiom.

Acknowledgments The author is in debt of Ori Haimanko for his patience, for his valuable remarks and suggestions, and for some invaluable discussions. The author also wishes to thank Dov Monderer for his helpful and valuable remarks, and to Benjamin Weiss for patiently answering many questions. Finally, I would like to thank Abraham Neyman and congratulate him on the occasion of his 66 birthday-Mazal Tov Merale!

Open Access This article is distributed under the terms of the Creative Commons Attribution 4.0 International License (http://creativecommons.org/licenses/by/4.0/), which permits unrestricted use, distribution, and reproduction in any medium, provided you give appropriate credit to the original author(s) and the source, provide a link to the Creative Commons license, and indicate if changes were made.

\section{Appendix 1: Proofs}

\section{Proof of Theorem 3}

\section{Marginals}

For $\lambda \in N A^{1}$ let $\mathcal{L} \mathcal{M}_{\lambda} \subseteq \mathcal{L} \mathcal{M}$ be the space of games of the form $f \circ \mu$ with $f \in L M^{k}$ and $\mu \in \mathcal{Z}_{\lambda}^{k}$ for some $k \geq 2$. Recall that the marginal ${ }^{8}$ is the function $\partial(f, \mu)$ defined in Eq. (7). Notice that $a \partial(f, \mu)=\partial(a f, \mu)$ for each $a \in \mathbb{R}$.

\footnotetext{
${ }^{8}$ It naturally depends on the representation of the game as $f \circ \mu$ of $f \circ \mu \in \mathcal{L} \mathcal{M}_{\lambda}$.
} 
We denote by $\partial \mathcal{L} \mathcal{M}_{\lambda}$ the linear space spanned by marginals of games $f \circ \mu$ with $f \in L M^{k}$ and $\mu \in \mathcal{Z}_{\lambda}^{k}$ for some $k$. Thus a typical element of $\partial \mathcal{L} \mathcal{M}_{\lambda}$ is of the form $\sum_{i=1}^{n} f_{i} \circ \mu^{i}$ with $f \in L M^{k_{i}}$ and $\mu^{i} \in \mathcal{Z}_{\lambda}^{k_{i}}$ for some $k_{1}, \ldots, k_{n}$. For $f \in L M^{k}$ and $\mu \in \mathcal{Z}_{\lambda}^{k}$ define the integral w.r.t. $\lambda$ by

$$
\int_{\lambda}(\partial(f, \mu))=f \circ \mu
$$

and extend $\int_{\lambda}$ to a linear map from $\partial \mathcal{L} \mathcal{M}_{\lambda}$ to $\mathcal{L} \mathcal{M}_{\lambda}$ by taking linear combination.

Remark 3 Note that $\int_{\lambda}$ is well defined. Indeed, if $g=\sum_{i=1}^{n} \partial\left(f_{i}, \mu^{i}\right)=$ $\sum_{j=1}^{m} \partial\left(h_{j}, v^{j}\right), f_{i} \in L M^{k_{i}}, h_{j} \in L M^{\ell_{j}}, \mu^{i} \in \mathcal{Z}_{\lambda}^{k_{i}}$, and $v^{i} \in \mathcal{Z}_{\lambda}^{\ell_{i}}$, let $F$ : $\bigotimes_{i=1}^{n} \mathbb{R}_{+}^{k_{i}} \rightarrow \mathbb{R}$ be given by $F\left(x^{1}, \ldots, x^{n}\right)=\sum_{i=1}^{n} f_{i}\left(x^{i}\right), k=\sum_{i=1}^{n} k_{i}$, and $\mu=\left(\mu^{1}, \ldots, \mu^{n}\right) \in \mathcal{Z}_{\lambda}^{k}$. Define a function $H, \ell \in \mathbb{N}$, and $v \in \mathcal{Z}_{\lambda}^{\ell}$, respectively, in a similar way. Let $G: \mathbb{R}_{+}^{k} \times \mathbb{R}_{+}^{\ell} \rightarrow \mathbb{R}$ be given by $G(x, y)=F(x)-H(y)$ and let $\eta=(\mu, v)$. Notice that $F \in L M^{k}, H \in L M^{\ell}$, and $G \in L M^{k+\ell}$. It is sufficient to prove that $G=0$ on the set $\mathcal{R}(\eta)$. Suppose first that $\eta(y) \in \mathcal{R}(\eta) \cap \mathbb{R}_{++}^{k+\ell}$ is a differentiability point of $G$, with $G$ being considered as a function on $\mathcal{R}(\eta)$, and $y \in B_{+}^{1}(T, \mathcal{C})$. We have

$$
\begin{aligned}
G \circ \eta(y) & =\nabla G(\eta(y)) \cdot \eta(y)=\int_{T} \nabla G(\eta(y)) \cdot \frac{d \eta}{d \lambda}(t) y(t) d \lambda(t) \\
& =\int_{T} d G\left(\eta(y), \frac{d \eta}{d \lambda}(t)\right) y(t) d \lambda(t) \\
& =\int_{T}\left(d F\left(\mu(y), \frac{d \mu}{d \lambda}(t)\right)-d H\left(v(y), \frac{d \nu}{d \lambda}(t)\right)\right) y(t) d \lambda(t) \\
& =\int_{T}\left(\sum_{i=1}^{n} d f_{i}\left(\mu^{i}(y), \frac{d \mu^{i}}{d \lambda}(t)\right)-\sum_{j=1}^{m} d h_{j}\left(v^{j}(y), \frac{d v^{j}}{d \lambda}(t)\right)\right) y(t) d \lambda(t) \\
& =\int_{T}\left(\sum_{i=1}^{n} \partial\left(f_{i}, \mu^{i}\right)(y)(t)-\sum_{j=1}^{m} \partial\left(h_{j}, v^{j}\right)(y)(t)\right) y(t) d \lambda(t)=0
\end{aligned}
$$

with the first equality in Line (30) following from the degree 1 homogeneity of $G$ and its differentiability at $\eta(y)$, the last equality in that line follows again from the differentiability of $G$ at $\eta(y)$, and the equality in Line (31) follows by Remark 1 as, since $\eta(y) \in \mathbb{R}_{++}^{k+\ell}, \mu^{i}(y) \neq 0$ and $\nu^{j}(y) \neq 0$ for $1 \leq i \leq n$ and $1 \leq j \leq m$ respectively. In particular, we obtain that $G=0$ on the set

$$
E(G, \eta)=\{z \in \mathcal{R}(\eta): G \text { is differentiable at } z\} \cap \mathbb{R}_{++}^{k+\ell} .
$$

The set $E(G, \eta)$ is dense in $\mathcal{R}(\eta)$ by Rademacher's theorem (applied for $G$ as a function $\mathcal{R}(\eta))$ as $G$ is a Lipschitz continuous function. The conclusion follows by the continuity of $G$. 
Remark 3 indicates that the integration mapping, or integral for short, $\int_{\lambda}$ is well defined.

Remark 4 Note that $\int_{\lambda}$ is also a positive mapping, namely, if $g \geq 0$ then $\int_{\lambda}(g)$ is monotonic. Let $g=\sum_{i=1}^{n} \partial\left(f_{i}, \mu^{i}\right) \geq 0$ and set $F$ and $\mu$ as in Remark 3. For fixed $y \leq y^{\prime}$ in $B_{+}^{1}(T, \mathcal{C})$ with $\mu(y) \supsetneqq \mu\left(y^{\prime}\right)$, there is a sequence $\left(z_{\ell}\right)_{\ell \in \mathbb{N}} \subseteq B_{+}^{1}(T, \mathcal{C})$ s.t. $\mu\left(z_{\ell}\right) \neq \mu(y)$ for each $\ell \geq 1, \lim _{\ell \rightarrow \infty} z_{\ell}=y^{\prime}$ in $L^{\infty}(\lambda)$, and $F$ is differentiable, as a function on $\mathcal{R}(\mu)$, for a.e. ${ }^{9} x \in\left[\mu(y), \mu\left(z_{\ell}\right)\right]$. Thus

$$
\begin{aligned}
F \circ \mu\left(y^{\prime}\right)-F \circ \mu(y)= & \lim _{\ell \rightarrow \infty}\left(F \circ \mu\left(z_{\ell}\right)-F \circ \mu(y)\right) \\
= & \lim _{\ell \rightarrow \infty} \int_{0}^{1} \nabla F\left(\mu(y)+s \mu\left(z_{\ell}-y\right)\right) \cdot \mu\left(z_{\ell}-y\right) d s \\
= & \lim _{\ell \rightarrow \infty} \int_{0}^{1} \int_{T} d F\left(\mu(y)+s \mu\left(z_{\ell}-y\right), \frac{d \mu}{d \lambda}(t)\right) \\
& \left(z_{\ell}(t)-y(t)\right) d \lambda(t) d s \\
= & \lim _{\ell \rightarrow \infty} \int_{0}^{1} \int_{T} g\left(y+s\left(z_{\ell}-y\right)\right)\left(z_{\ell}(t)-y(t)\right) d \lambda(t) d s \\
= & \lim _{\ell \rightarrow \infty}\left(\int_{0}^{1} \int_{T} g\left(y+s\left(z_{\ell}-y\right)\right)\left(y^{\prime}(t)-y(t)\right) d \lambda(t) d s\right. \\
& \left.+\int_{0}^{1} \int_{T} g\left(y+s\left(z_{\ell}-y\right)\right)\left(z_{\ell}(t)-y^{\prime}(t)\right) d \lambda(t) d s\right) \\
\geq & \lim _{\ell \rightarrow \infty} \int_{0}^{1} \int_{T} g\left(y+s\left(z_{\ell}-y\right)\right)\left(z_{\ell}(t)-y^{\prime}(t)\right) d \lambda(t) d s=0,
\end{aligned}
$$

with the equality in Line (33) following from Remark 1 , as for a.e. $s \in[0,1]$ we have $0 \neq \mu(y)+s \mu\left(z_{\ell}-y\right)$ for every $\ell$, the inequality in Line (32) follows as $g \geq 0$ and $y^{\prime}-y \geq 0$, and the equality in Line (33) by the dominated convergence theorem as the sequence of functions $h_{\ell}:(s, t) \mapsto g\left(y+s\left(z_{\ell}-y\right)\right)\left(z_{\ell}(t)-y^{\prime}(t)\right)$ is bounded and converges to 0 , for $s \otimes \lambda$-a.e. $(s, t) \in[0,1] \times T$, as $\ell \rightarrow \infty$. The claim follows.

We can conclude this Section with the following Lemma:

Lemma 4 The integral $\int_{\lambda}$ is a well defined positive linear map.

The proof

Lemma 5 If $v \in \mathcal{L} \mathcal{M}_{\lambda}$ then $\varphi(v) \ll \lambda$ and $\frac{d \varphi(v)}{d \lambda} \in L^{2}(\lambda)$.

Proof As $v+a \lambda$ is monotonic for any large enough $a>0$, and $\varphi(a \lambda)=a \lambda$, it is sufficient to prove the Lemma for a monotonic game $v \in \mathcal{L} \mathcal{M}_{\lambda}$. Choose $K_{v}>0$ s.t.

9 W.r.t. the Lebesgue measure on the interval. 
$w \equiv K_{v} \lambda-v$ is monotonic. Therefore, as $v$ is monotonic and $\varphi\left(K_{v} \lambda\right)=K_{v} \lambda$, we obtain, obtain (inequalities are in the $B V$ sense)

$$
0 \leq \varphi(v) \leq K_{v} \lambda
$$

Hence $\varphi(v) \ll \lambda$ and

$$
0 \leq \frac{d \varphi(v)}{d \lambda} \leq K_{v}
$$

where the inequalities above hold in $L^{1}(\lambda)$.

The operator $\psi_{\lambda}: \partial L M_{\lambda} \rightarrow L^{2}(\lambda)$ given by

$$
\psi_{\lambda}(g)=\frac{d \varphi\left(\int_{\lambda}(g)\right)}{d \lambda},
$$

is well defined. As the maps $\int_{\lambda}$ (by Lemma 4) and $\varphi$ are linear and positive then so is $\psi_{\lambda}$. By definition, the constant functions are contained in $\partial \mathcal{L} \mathcal{M}_{\lambda}$, which makes it a massive ${ }^{10}$ subspace of $B\left(X_{\lambda}, L^{\infty}(\lambda)\right)$ as every $g \in \partial \mathcal{L} \mathcal{M}_{\lambda}$ is bounded by $\|g\|_{\infty}$. Therefore, by Kantorovich's theorem (Theorem 5, in the Appendix) $\psi_{\lambda}$ extends to a positive linear operator $\Psi_{\lambda}: B\left(X_{\lambda}, L^{\infty}(\lambda)\right) \rightarrow L^{2}(\lambda)$.

Restricting our attention to the subspace $C\left(X_{\lambda}, L^{\infty}(\lambda)\right) \subseteq B\left(X_{\lambda}, L^{\infty}(\lambda)\right)$, then by the Dinculeanu-Singer theorem (Theorem 7 in the Appendix) we obtain ${ }^{11}$ that there is a unique ${ }^{12}$ positive, finitely additive Borel vector measure $P_{\lambda}$ on $X_{\lambda}$ of bounded semi-variation with values in $\mathcal{L}\left(L^{\infty}(\lambda), L^{2}(\lambda)\right)$, s.t. for every $g \in C\left(X_{\lambda}, L^{\infty}(\lambda)\right)$

$$
\Psi_{\lambda}(g)=\int_{X_{\lambda}} g(x) d P_{\lambda}(x) .
$$

By Remark 7 (in the Appendix) the positivity of the operator $\Psi_{\lambda}$ yields the positivity of the vector measure $P_{\lambda}$. By property (i) of the Dinculeanu-Singer theorem (Theorem 7 in the Appendix) for every $S \in \mathcal{C}$ the vector measure $P_{\lambda}^{S}=\left\langle P_{\lambda}, \chi_{S}\right\rangle$ is a positive, regular, and countably additive vector measure on the Borel subsets of $X_{\lambda}$ with values in $\left.\mathcal{L}\left(L^{\infty}(\lambda), \mathbb{R}\right)\right) \cong\left(L^{\infty}(\lambda)\right)^{*}$, and by definition it has a bounded variation. Now, if $g \in C\left(X_{\lambda}, L^{\infty}(\lambda)\right) \cap \partial \mathcal{L} \mathcal{M}_{\lambda}$ and $S \in \mathcal{C}$ then

\footnotetext{
10 See Appendix 3 for the definition.

11 Notice that in this case every positive linear operator $A: C\left(X_{\lambda}, L^{\infty}(\lambda)\right) \rightarrow L^{2}(\lambda)$ is bounded; indeed, for every $f \in C\left(X_{\lambda}, L^{\infty}(\lambda)\right)$ and every $x \in X_{\lambda}$ we have $-\|f\|_{\infty} \leq f(x) \leq\|f\|_{\infty}$, thus $-\|f\|_{\infty} \leq$ $f \leq\|f\|_{\infty}$. Now $A(f)$ is a member of the Banach lattice $L^{2}(\lambda)$. By the positivity of $A$ we obtain $|A(f)| \leq\|f\|_{\infty}|A(1)|$ in the Banach lattice $L^{2}(\lambda)$ and therefore $\|A(f)\|_{2} \leq\|A(1)\|_{2}\|f\|_{\infty}$. Hence $A$ is bounded.

12 W.r.t. $\Psi_{\lambda}, \operatorname{not} \psi_{\lambda}$.
} 


$$
\begin{aligned}
\varphi\left(\int_{\lambda}(g)\right)(S) & =\int_{S} \psi_{\lambda}(g)(s) d \lambda(s) \\
& =\int_{S}\left(\int_{X_{\lambda}} g(x) d P_{\lambda}(x)\right)(s) d \lambda(s)=\int_{X_{\lambda}} g(x) d P_{\lambda}^{S}(x)
\end{aligned}
$$

where the first equality in line (36) follows from the definition of $\psi_{\lambda}$, the second equality in that line follows from Eq. (35), and the equality in line (37) follows from property (iii) of the Dinculeanu-Singer theorem (Theorem 7 in the Appendix). This proves Eq. (10).

Remark 5 A function $g \in \partial \mathcal{L} \mathcal{M}_{\lambda}$ is weakly lower semi-continuous iff there is a bounded sequence $\left(g^{n}\right)_{n=1}^{\infty} \subseteq C\left(X_{\lambda}, L^{\infty}(\lambda)\right)$, s.t. $g^{n} \leq g$ for every $n \geq 1$, and $g^{n} \underset{n \rightarrow \infty}{\longrightarrow} g$ pointwise. By the positivity of $\Psi_{\lambda}$ we have in this case

$$
\begin{aligned}
\Psi_{\lambda}\left(g^{n}\right) & \leq \Psi_{\lambda}(g) \Rightarrow \\
\forall S \in \mathcal{C}, \quad \int_{X_{\lambda}} g^{n}(x) d P_{\lambda}^{S} & \leq \int_{S} \Psi_{\lambda}(g)(s) d \lambda(s)
\end{aligned}
$$

As $g \in \partial \mathcal{L} \mathcal{M}_{\lambda}$ we have $\Psi_{\lambda}(g)=\psi_{\lambda}(g)$, hence by definition $\int_{S} \Psi_{\lambda}(g)(s) d \lambda(s)=$ $\varphi\left(\int_{\lambda}(g)\right)(S)$ and hence

$$
\varphi\left(\int_{\lambda}(g)\right)(S) \geq \int_{X_{\lambda}} g(x) d P_{\lambda}^{S}(x)
$$

Remark 6 Notice that $\partial(f, \mu)$ is weakly lower semi-continuous for every $f \in L M_{+}^{k}$ and $\mu \in \mathcal{Z}_{\lambda}^{k}$. Indeed, let $K \subseteq \mathbb{R}_{+}^{k}$ be a compact set with $\frac{d \mu}{d \lambda} \in K \lambda$-a.e. It is an immediate consequence from (Rockafellar 1970, Theorems 24.5, 24.6 ) that the function $g_{\mu}(f)$ on $\Lambda_{\mu} \times K$ given by $g_{\mu}(f)(x, y)=\left\{\begin{array}{ll}d f(x, y), & x \in \Delta(\mu) \\ d f\left(\mathbf{1}_{k}, x, y\right), & x \in \mathbb{S}_{\perp}^{\mu}\end{array}\right.$ is lower semi-continuous. The metric on $\Lambda_{\mu} \times K$ is equivalent to the metric given by $\eta\left((x, y),\left(x^{\prime}, y^{\prime}\right)\right)=d\left(x, x^{\prime}\right)+\left\|y-y^{\prime}\right\|_{2}$ with $d$ being a metric on $\Lambda_{\mu}$. By (Aliprantis and Border 2006, Theorem 3.13) there is an increasing and bounded sequence of Lipschitz ${ }^{13}$ continuous functions $\left(g^{n}\right)_{n=1}^{\infty} \subseteq C\left(\Lambda_{\mu} \times K\right)$, s.t. $g^{n} \leq g_{\mu}(f)$ for every $n \geq 1$, and $g^{n} \underset{n \rightarrow \infty}{\longrightarrow} g_{\mu}(f)$ pointwise. For $x \in X_{\lambda}$, define $G^{n}(x)=g^{n}\left(x(\mu), \frac{d \mu}{d \lambda}\right)$. For each $n \geq 1$ we have $G^{n}(x) \in L^{\infty}(\lambda)$ as $g^{n}$ is bounded on $\Lambda_{\mu} \times K$. Furthermore, $G^{n} \in C\left(X_{\lambda}, L^{\infty}(\lambda)\right)$ for each $n \geq 1$. Indeed, if $M^{n}$ denotes the Lipschitz constant of $g^{n}$ w.r.t. the metric $\eta$, and $\left(x^{\beta}\right)_{\beta} \subseteq X_{\lambda}$ is a net converging to $x \in X_{\lambda}$ then

$$
\begin{aligned}
\left\|G^{n}(x)-G^{n}\left(x^{\beta}\right)\right\|_{\infty} & =\left\|g^{n}\left(x(\mu), \frac{d \mu}{d \lambda}\right)-g^{n}\left(x^{\beta}(\mu), \frac{d \mu}{d \lambda}\right)\right\|_{\infty} \\
& \leq M^{n} d\left(x(\mu), x^{\beta}(\mu)\right) \underset{\beta}{\rightarrow} 0,
\end{aligned}
$$

13 W.r.t. $\eta$. 
and the continuity of $G^{n}$ follows. Finally, as the sequence $\left(g^{n}\right)_{n \in \mathbb{N}}$ is increasing and the function $g_{\mu}(f)(x, \cdot): y \mapsto g_{\mu}(f)(x, y)$ is continuous for every $x \in \Lambda_{\mu}$, then by Dini's theorem $g^{n}(x, \cdot) \underset{n \rightarrow \infty}{\longrightarrow} g_{\mu}(f)(x, \cdot)$ uniformly on $K$ for each $x \in \Lambda_{\mu}$. Thus, in $L^{\infty}(\lambda)$,

$$
\lim _{n \rightarrow \infty} G^{n}(x)=\lim _{n \rightarrow \infty} g^{n}\left(x(\mu), \frac{d \mu}{d \lambda}\right)=g_{\mu}(f)\left(x(\mu), \frac{d \mu}{d \lambda}\right)=\partial(f, \mu)(x)
$$

for each $x \in X_{\lambda}$, which proves that $\partial(f, \mu)$ is weakly lower semi-continuous for every $f \in L M_{+}^{k}$ and $\mu \in \mathcal{Z}_{\lambda}^{k}$.

To prove Eq. (9), we shall consider the set $\mathcal{F}$ of operators extending $\psi_{\lambda}$ to a positive linear operator $\phi: B\left(X_{\lambda}, L^{\infty}(\lambda)\right) \rightarrow L^{2}(\lambda)$. Notice that every $\phi \in \mathcal{F}$ is bounded with norm ${ }^{14} 1$, thus $\mathcal{F}$ is norm bounded. It is also a closed subset, in the operator weak $^{*}$ topology ${ }^{15}$ of the space $\mathcal{O}_{\lambda}$ of bounded linear operators from $B\left(X_{\lambda}, L^{\infty}(\lambda)\right)$ to $L^{2}(\lambda)$. Hence, by Theorem 8 (in the Appendix) we deduce that $\mathcal{F}$ is also compact in this topology. Furthermore $\mathcal{F}$ is convex.

Let $\theta \in \Theta$ be $\lambda$-preserving and mixing, and denote by $G$ the cyclic group generated by $\theta$. For $x \in X_{\lambda}$ and $\tau \in G$ let $\tau x \in X_{\lambda}$ be given by $\tau x(\mu)=x(\tau \mu)$, and define a linear mapping from $B\left(X_{\lambda}, L^{\infty}(\lambda)\right)$ to itself by $A_{\tau}(g)=g(\tau x) \circ \tau$. Consider the group action of $G$ on $\mathcal{O}_{\lambda}$ given by

$$
\forall \tau \in G, \phi \in \mathcal{O}_{\lambda}, h \in B\left(X_{\lambda}, L^{\infty}(\lambda)\right), \quad(\tau, \phi)(h)=\phi\left(A_{\tau}(h)\right) \circ \tau^{-1}
$$

This group action maps $\mathcal{F}$ to itself. Indeed for every $\tau \in G$ and $\phi \in \mathcal{F},(\tau, \phi)$ is a positive linear operator from $B\left(X_{\lambda}, L^{\infty}(\lambda)\right)$ to $L^{2}(\lambda)$, and for every $g \in \partial \mathcal{L} \mathcal{M}_{\lambda}$ we have

$$
\begin{aligned}
(\tau, \phi)(g) & =\phi\left(A_{\tau}(g)\right) \circ \tau^{-1}=\psi_{\lambda}\left(A_{\tau}(g)\right) \circ \tau^{-1}=\frac{d \varphi\left(\int_{\lambda}\left(A_{\tau}(g)\right)\right)}{d \lambda} \circ \tau^{-1} \\
& =\frac{d \varphi\left(\tau^{*} \int_{\lambda}(g)\right)}{d \lambda} \circ \tau^{-1}=\frac{d \varphi\left(\int_{\lambda}(g)\right)}{d \lambda}=\psi_{\lambda}(g),
\end{aligned}
$$

where the last equality in line (40) follows as, by assumption, $\int_{\lambda}\left(A_{\tau}(g)\right)=\tau^{*} \int_{\lambda}(g)$ and the first equality in line (41) follows from the symmetry axiom. Hence, $G(\mathcal{F}) \subseteq \mathcal{F}$. Notice now that for $\tau \in G$ the map $\phi \mapsto(\tau, \phi)$ defined on $\mathcal{O}_{\lambda}$ is continuous. Indeed, if $\phi_{\beta} \underset{\beta \in B}{\longrightarrow} \phi$ is a net in $\mathcal{O}_{\lambda}$ converging to $\phi \in \mathcal{O}_{\lambda}$ in the weak* operator topology, then

\footnotetext{
14 Indeed, we have $\|\phi(g)\|_{2} \leq\|\phi(1)\|_{2}\|g\|_{\infty}$. Since $\phi(1)=\psi_{\lambda}(1)=1$ then $\|\phi\|=1$.

15 Namely, the weakest topology on $\mathcal{O}_{\lambda}$ s.t. the maps
}

$$
\phi \stackrel{g}{\mapsto} \phi(g), \quad \phi \in \mathcal{O}_{\lambda}, g \in B\left(X_{\lambda}, L^{\infty}(\lambda)\right)
$$

are continuous for every $g \in B\left(X_{\lambda}, L^{\infty}(\lambda)\right)$. 
for every $h \in B\left(X_{\lambda}, L^{\infty}(\lambda)\right)$ and $\chi \in L^{2}(\lambda)$ we have $\left\langle\left(\phi_{\beta}-\phi\right)(h), \chi\right\rangle \underset{\beta \in B}{\longrightarrow} 0$, hence

$$
\begin{aligned}
\left\langle\left((\tau, \phi)-\left(\tau, \phi_{\beta}\right)\right)(h), \chi\right\rangle & =\left\langle\left(\phi-\phi_{\beta}\right)\left(A_{\tau}(h)\right) \circ \tau^{-1}, \chi\right\rangle \\
& =\left\langle\left(\phi-\phi_{\beta}\right)\left(A_{\tau}(h)\right), \chi \circ \tau\right\rangle \underset{\beta \in B}{\longrightarrow} 0 .
\end{aligned}
$$

We thus proved that the action of $G$ induces a commuting family of continuous linear mappings on $\mathcal{O}_{\lambda}$ which maps its compact and convex subset $\mathcal{F}$ to itself. Hence by Markov-Kakutani fixed point theorem (Dunford and Schwartz 1957, p. 456, Theorem 6) there is some $\phi_{0} \in \mathcal{F}$ with $\left(\tau, \phi_{0}\right)=\phi_{0}$ for every $\tau \in G$. By perhaps amending the choice of the extension $\Psi_{\lambda}$, by setting $\Psi_{\lambda}=\phi_{0}$, and letting $P_{\lambda}$ be the representing measure of the restriction of this operator to $C\left(X_{\lambda}, L^{\infty}(\lambda)\right.$ ) (given by Eq. 35), our proof of Eq. (10) holds. In fact, for every $g \in C\left(X_{\lambda}, L^{\infty}(\lambda)\right)$, every $S \in \mathcal{C}$, and every ${ }^{16}$ $\tau \in G$ we thus have

$$
\int_{X_{\lambda}} g(x) d P_{\lambda}^{\tau S}(x)=\int_{X_{\lambda}} A_{\tau}(g)(x) d P_{\lambda}^{S}(x) .
$$

For $h \in B\left(X_{\lambda}, L^{\infty}(\lambda)\right)$ take a uniformly bounded sequence $\left(g_{m}\right)_{m=1}^{\infty} \subseteq C\left(X_{\lambda}, L^{\infty}(\lambda)\right)$ converging $\left\langle 1, P_{\lambda}^{T}\right\rangle$-a.e. to $h$. By applying the bounded convergence theorem (Theorem 6 in the Appendix) to Eq. (43) we obtain for every $\tau \in G$ and $S \in \mathcal{C}$ as $m \rightarrow \infty$

$$
\int_{X_{\lambda}} h(x) d P_{\lambda}^{\tau S}(x)=\int_{X_{\lambda}} A_{\tau}(h)(x) d P_{\lambda}^{S}(x) .
$$

Setting $\bar{\Psi}_{\lambda}^{S}=\int_{X_{\lambda}} h(x) d P_{\lambda}^{S}(x)$ we obtain

$$
\bar{\Psi}_{\lambda}^{\tau S}=\bar{\Psi}_{\lambda}^{S} \circ A_{\tau}
$$

We shall now prove that $\bar{\Psi}$ coincides with $\psi_{\lambda}$ on $\partial \mathcal{L} \mathcal{M}_{\lambda}$ which will complete the proof of the theorem.

Lemma 6 For every $f \in L M^{k}$ and $\mu \in \mathcal{Z}_{\lambda}^{k}$ we have

$$
\bar{\Psi}_{\lambda}^{T}(\partial(f, \mu))=f(\mu(T))
$$

Proof It is sufficient to prove the equality for $f \in L M_{+}^{k}$, which will be assumed henceforth. We have

$$
f\left(\mathbf{1}_{k}\right)=\Psi_{\lambda}^{T}(\partial(f, \mu)) \geq \bar{\Psi}_{\lambda}^{T}(\partial(f, \mu))
$$

with the first equality in Line (48) following from the efficiency axiom for the first and the second inequality following by combining Remarks 5 and 6 . We thus need

16 If $\tau$ is the ID map, we obtain Eq. (10). 
to prove the inverse inequality. For every Borel set $E \subseteq X_{\lambda}$ and $\phi \in L^{\infty}(\lambda)$ with $\int_{T} \phi(s) d \lambda(s)=1$ we have

$$
\begin{aligned}
\left\langle\phi \circ \theta^{n}, P_{\lambda}^{T}\right\rangle(E)= & \int_{T} \phi\left(\theta^{n}(s)\right)\left\langle 1, P_{\lambda}\right\rangle(E)(s) d \lambda(s) \underset{n \rightarrow \infty}{\longrightarrow} \\
& \left(\int_{T} \phi(s) d \lambda(s)\right)\left(\int_{T}\left\langle 1, P_{\lambda}\right\rangle(E)(s) d \lambda(s)\right)=\left\langle 1, P_{\lambda}^{T}\right\rangle(E),
\end{aligned}
$$

where the equality in the display (47) follows by combining Lemma 1 with the definition of $P_{\lambda}^{T}$, and the limit follows as $\theta$ is strongly mixing. Hence, by Scheefe's Lemma (Williams 1991, Lemma 5.10, p. 55), for every $1 \leq i \leq k$ the sequence of measures $\left(v_{n}^{i}=\left\langle\frac{d \mu_{i}}{d \lambda} \circ \theta^{n}-1, P_{\lambda}^{T}\right\rangle\right)_{n=1}^{\infty}$ converges to 0 in variation ${ }^{17}$. Also notice that by the concavity and monotonicity of $f$ we may write

$$
\partial(f, \mu)(x) \geq f\left(\mathbf{1}_{k}\right)+\sum_{i=1}^{k} g_{i}(x)\left(\frac{d \mu_{i}}{d \lambda}-1\right)
$$

for every $x \in X_{\lambda}$, where $g_{i}: X_{\lambda} \rightarrow \mathbb{R}_{+}$is bounded for every $1 \leq i \leq k$. Indeed,

$$
\begin{aligned}
d f\left(x, \mathbf{1}_{k}\right) & =\lim _{\epsilon \rightarrow 0^{+}} \frac{1}{\epsilon}\left(f\left(x+\epsilon \mathbf{1}_{k}\right)-f(x)\right) \\
& \geq \lim _{\epsilon \rightarrow 0^{+}} \frac{1}{\epsilon}\left(f(x)+\epsilon f\left(\mathbf{1}_{k}\right)-f(x)\right)=f\left(\mathbf{1}_{k}\right),
\end{aligned}
$$

with the last inequality in Line (49) following by combining the concavity and degree 1 homogeneity of $f$. Applying same reasoning to the degree 1 homogeneous concave function $d f\left(\mathbf{1}_{k}, \cdot\right)$ we obtain

$$
d f\left(\mathbf{1}_{k}, x, \mathbf{1}_{k}\right) \geq d f\left(\mathbf{1}_{k}, \mathbf{1}_{k}\right)=f\left(\mathbf{1}_{k}\right) .
$$

Now, if $x \in \Delta(\mu)$ we have,

$$
\begin{aligned}
d f\left(x, \frac{d \mu}{d \lambda}\right) & \geq d f\left(x, \frac{d \mu}{d \lambda}-\mathbf{1}_{k}\right)+d f\left(x, \mathbf{1}_{k}\right) \geq d f\left(x, \frac{d \mu}{d \lambda}-\mathbf{1}_{k}\right)+f\left(\mathbf{1}_{k}\right) \\
& \geq f\left(\mathbf{1}_{k}\right)+\sum_{i=1}^{k} d f\left(x, e_{i}\right)\left(\frac{d \mu_{i}}{d \lambda}-1\right)
\end{aligned}
$$

with the first and last inequalities in Line (52) following from the concavity of $d f(x, \cdot)$. Thus, in this case we choose $g_{i}(x)=d f\left(x, e_{i}\right)$. We can repeat that for $x \in \mathbb{S}_{\perp}^{\mu}$, and in that case choose $g_{i}(x)=d f\left(\mathbf{1}_{k}, x, e_{i}\right)$.

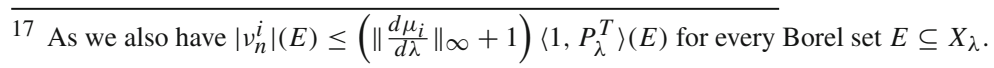


Therefore, for every $n \geq 1$

$$
\begin{aligned}
\bar{\Psi}_{\lambda}^{T}(\partial(f, \mu)) & =\int_{X_{\lambda}} A_{\theta^{n}}(\partial(f, \mu))(x) d P_{\lambda}^{T}(x) \\
& \geq f\left(\mathbf{1}_{k}\right)+\sum_{i=1}^{k} \int_{X_{\lambda}} g_{i}\left(\theta^{n} x\right) d\left\langle\left(\frac{d \mu_{i}}{d \lambda} \circ \theta^{n}-1\right), P_{\lambda}^{T}\right\rangle(x) \\
& =f\left(\mathbf{1}_{k}\right)+\sum_{i=1}^{k} \int_{X_{\lambda}} g_{i}\left(\theta^{n} x\right) d v_{n}^{i}(x)
\end{aligned}
$$

where the second equality in line (54) follows from Eq. (44) as $\theta^{n} T=T$ and the next inequality follows by combining Eq. (48) with the positivity of the vector measure $P_{\lambda}^{T}$. The Lemma now follows by taking $n \rightarrow \infty$ in Eq. (55), as for every $1 \leq i \leq k$ the function $g_{i}$ is bounded on $X_{\lambda}$ and $\nu_{n}^{i} \underset{n \rightarrow \infty}{\longrightarrow} 0$ in variation.

Finally, for every $f \in L M^{k}, \mu \in \mathcal{Z}_{\lambda}^{k}$, and $S \in \mathcal{C}$

$$
\begin{aligned}
f\left(\mathbf{1}_{k}\right) & =\varphi(f \circ \mu)(T)=\varphi(f \circ \mu)(S)+\varphi(f \circ \mu)\left(S^{c}\right) \\
& \geq \int_{X_{\lambda}} \partial(f, \mu)(x) d P_{\lambda}^{S}(x)+\int_{X_{\lambda}} \partial(f, \mu)(x) d P_{\lambda}^{S^{c}}(x) \\
& =\int_{X_{\lambda}} \partial(f, \mu)(x) d P_{\lambda}^{T}(x)=f\left(\mathbf{1}_{k}\right),
\end{aligned}
$$

with the first equality in Line (56) following from the efficiency axiom, the last inequality in that line following from Eq. (38), and the last equality in line (57) following from Lemma 6. With Eq. (38) in mind we deduce

$$
\varphi(f \circ \mu)(S)=\int_{X_{\lambda}} \partial(f, \mu)(x) d P_{\lambda}^{S}(x),
$$

and the theorem follows.

\section{Proof of lemma 1}

For $\phi \in L^{\infty}(\lambda)$ and $E \in \mathcal{B}\left(X_{\lambda}\right),\left\langle\phi, P_{\lambda}\right\rangle(E) \equiv P_{\lambda}(E)(\phi)$, and $P_{\lambda}(E) \in$ $\mathcal{L}\left(L^{\infty}(\lambda), L^{2}(\lambda)\right)$. If $\left\|P_{\lambda}(E)\right\| \|$ denotes the operator norm, then $\left\|P_{\lambda}(E)(\phi)\right\|_{2} \leq$ $\|\phi\|_{\infty}\left\|P_{\lambda}(E)\right\| \|$, so $\left\langle\phi, P_{\lambda}\right\rangle(E) \rightarrow 0$ in $L^{2}(\lambda)$ as $\phi \rightarrow 0$ in $L^{\infty}(\lambda)$. As the simple functions are dense in $L^{\infty}(\lambda)$ and $\phi \mapsto\left\langle\phi, P_{\lambda}\right\rangle(E)$ is linear, it is sufficient to prove the Lemma for characteristic functions.

For every $S \in \mathcal{C}$ we have

$$
\left\langle\chi_{S}, P_{\lambda}\right\rangle(E)=\left\langle 1, P_{\lambda}\right\rangle(E) \chi_{S} .
$$


Indeed, for every $S, S^{\prime} \in \mathcal{C}$ with $\lambda\left(S^{\prime} \cap S\right)=0$ we have

$$
\begin{array}{r}
0 \leq\left\langle\chi_{S}, P_{\lambda}^{S^{\prime}}\right\rangle(E) \leq\left\langle\chi_{S}, P_{\lambda}^{S^{\prime}}\right\rangle\left(X_{\lambda}\right)=\int \chi_{S}(s) \chi_{S^{\prime}}(s) d \lambda(s)=0 \Rightarrow \\
\left\langle\chi_{S}, P_{\lambda}\right\rangle(E)=\left\langle\chi_{S}, P_{\lambda}\right\rangle(E) \chi_{S},
\end{array}
$$

where the second inequality in line (59) above follows from the positivity of $P_{\lambda}$ and following equality follows as $\Psi_{\lambda}$ is a projection. Hence

$$
\left\langle 1, P_{\lambda}\right\rangle(E) \chi_{S}=\left\langle\chi_{S}, P_{\lambda}\right\rangle(E) \chi_{S}+\left\langle\chi_{S^{c}}, P_{\lambda}\right\rangle(E) \chi_{S}=\left\langle\chi_{S}, P_{\lambda}\right\rangle(E),
$$

and the Lemma follows.

\section{Appendix 2: The Mertens value on $\mathcal{M}$}

Here we briefly describe the form of the Mertens value on $\mathcal{M}$. For a detailed construction, see Mertens (1988).

For $\mu \in\left(N A^{1}\right)^{k}$, let

$$
\|y\|_{\mu}=\int\left|\sum_{i=1}^{k}\left(d \mu_{i} / d \bar{\mu}\right) y_{i}\right| d \bar{\mu}
$$

with $\bar{\mu}=\frac{1}{k} \sum_{i=1}^{k} \mu_{i}$. By (Neyman 2001, Lemma 1 ) the function $\phi_{\mu}: A F(\mu) \longrightarrow \mathbb{R}$ given by $\phi_{\mu}(y)=\exp \left(-\|y\|_{\mu}\right)$ is the characteristic function of a probability distribution $P_{\mu}$. Denote by $Q_{\mu}$ the push-forward measure of $P_{\mu}$ w.r.t. the mapping ${ }^{18}$ $z \mapsto \frac{z-\bar{z} \mathbf{1}_{k}}{\left\|z-\bar{z} \mathbf{1}_{k}\right\|_{2}}$. This is a measure on $\mathbb{S}_{\perp}^{\mu}$.

The Mertens value on $\mathcal{M}$ is given, for $f \in M^{k}, \mu \in\left(N A^{1}\right)^{k}$, and $S \in \mathcal{C}$, by (see Mertens 1988)

$$
\varphi_{M}(f \circ \mu)(S)=\int d f\left(\mathbf{1}_{k}, x, \mu(S)\right) d Q_{\mu}(x) .
$$

\section{Appendix 3: Rudiments of functional analysis}

Here we give some functional analysis background which is needed for the understanding of some of our results. For further reading, one is advised to use the reference.

\section{Extension of linear operators}

A Banach lattice $Z$ is a Banach space which is a lattice, and if $0 \leq x \leq y$ then $\|x\| \leq\|y\|$. A Banach lattice is a $K$-space iff it is order complete, i.e., if every

18 We use the convention $\frac{0}{0}=0$. 
nonempty and bounded from above (below) $A \subseteq Z$ has a least (greatest) upper (lower) bound.

If $X$ is a Banach lattice then $X^{*}$ with its positive cone

$$
X_{+}^{*}=\left\{x^{*} \in X^{*}: \forall x \in X_{+}, x^{*}(x) \geq 0\right\}
$$

is a $K$-space (see Bukhvalov et al. 1979, p. 162), and hence every reflexive Banach lattice is a $K$-space. Hence, for every $\lambda \in N A^{1}, L^{p}(\lambda)$ is a $K$-space for every $1<$ $p \leq \infty$.

A subspace $V$ of a Banach lattice $Z$ is massive iff for every $z \in Z$ there is a $v \in V$ s.t. $z \leq v$.

Theorem 5 (Kantorovich) (Kusraev 2000, Theorem 3.1.7) Let $Z$ be a Banach lattice and $Y$ a $K$-space. Then if $V$ is a massive subspace of $Z$ and $T: V \rightarrow Y$ is a positive linear operator then $T$ can be extended to a positive linear operator $\bar{T}: Z \rightarrow Y$.

\section{Vector measures}

A function $F$ from an algebra $\mathfrak{F}$ of subsets of a set $\Omega$ to a Banach space $Z$ is called finitely additive vector measure or simply a vector measure if whenever $E_{1}, E_{2} \in \mathfrak{F}$ are disjoint then $F\left(E_{1} \cup E_{2}\right)=F\left(E_{1}\right)+F\left(E_{2}\right)$. If, in addition, $F\left(\bigcup_{n=1}^{\infty} E_{n}\right)=$ $\sum_{n=1}^{\infty} F\left(E_{n}\right)$ in the norm topology of $Z$ for all sequences $\left(E_{n}\right)_{n=1}^{\infty}$ of pairwise disjoint members of $\mathfrak{F}$ s.t. $\bigcup_{n=1}^{\infty} E_{n} \in \mathfrak{F}$ then $F$ is termed a countably additive vector measure or simply countably additive.

The strong variation of $F$ is the function $\|F\|: \mathfrak{F} \rightarrow \mathbb{R}$ defined by

$$
\|F\|(E)=\sup _{\pi} \sum_{A \in \pi}\|F(A)\|
$$

where the supremum is taken over all finite partitions of $E$ into disjoint members of $\mathfrak{F}$. One may easily check that $\|F\|$ is a monotonic finitely additive measure. A measure $F$ is of bounded variation if $\|F\|(\Omega)<\infty$. Furthermore,

Proposition 2 (Diestel and Uhl Jr 1977, Proposition I.1.9) A vector measure of bounded variation is countably additive iff its variation is also countably additive.

\section{Integration w.r.t. a measure with values in $\mathcal{L}(Y, Z)$}

Let $F$ be a vector measure on an algebra $\mathfrak{F}$ of subsets of $\Omega$ with values in the Banach space $\mathcal{L}(Y, Z)$ of bounded linear operators from $Y$ to $Z$, where $Y, Z$ are Banach lattices. Denote by $\mathcal{S}_{\Omega, \mathfrak{F}}(Y)$ the set of simple functions on $\Omega$ w.r.t. $\mathfrak{F}$ taking values in $Y$, i.e. the set of functions of the form $\sum_{i=1}^{n} a_{i} \chi_{E_{i}}$ where $E_{i} \in \mathfrak{F}$ and $a_{i} \in Y$ for every $1 \leq i \leq n$. The (Bartle) integral of such a simple $f=\sum_{i=1}^{n} a_{i} \chi_{E_{i}}$ w.r.t. $F$ is given 
by

$$
\int f d F=\sum_{i=1}^{n} F\left(E_{i}\right)\left(a_{i}\right) .
$$

A measurable function $f: \Omega \rightarrow Y$ is strongly $F$-integrable, or integrable for short, if for every increasing sequence $\left(f_{n}\right)_{n=1}^{\infty}$ of simple functions $f_{n}: \Omega \rightarrow Y$ with $f_{n} \underset{n \rightarrow \infty}{\longrightarrow} f$ pointwise $\|F\|$-a.e. the limit $\nu(E)=\lim _{n \rightarrow \infty} \int f_{n} \chi_{E} d F$ exists in the strong topology of $Z$ for every $E \in \mathfrak{F}$ and is independent of the choice of $\left(f_{n}\right)_{n=1}^{\infty}$. In that case we denote

$$
\int_{E} f d F=\lim _{n \rightarrow \infty} \int_{E} f_{n} d F .
$$

Theorem 6 (Bartle bounded convergence theorem) (Diestel and Uhl Jr 1977, Theorem 1, p. 56) Let $\left(f_{n}\right)_{n=1}^{\infty}$ be a uniformly bounded sequence of integrable functions $f_{n}: \Omega \rightarrow Y$, and suppose that $F$ above is countably additive of bounded variation. If $\left(f_{n}\right)$ converges $\|F\|$-a.e. to $f$ then $f$ is integrable and

$$
\lim _{n \rightarrow \infty} \int f_{n} d F=\int f d F
$$

in the strong topology of $Z$.

\section{Representation of bounded linear operators}

Let $Z, Y$ be Banach spaces, $\Omega$ a compact and Hausdorff space. If $G$ is a measure on the Borel $\sigma$-algebra $\mathcal{B}_{\Omega}$ of $\Omega$ taking values in $\mathcal{L}\left(Y, Z^{* *}\right)$ then for every $z^{*} \in Z^{*}$ we define the measure $G_{z^{*}}: \mathcal{B}_{\Omega} \rightarrow Y^{*}$ by $G_{z^{*}}(A)(y)=\left\langle G(A)(y), z^{*}\right\rangle$ where $\langle\cdot, \cdot\rangle$ is the usual pairing. The semi-variation $|G|(E)$ of $G$ on $E \in \mathcal{B}_{\Omega}$ is given by $|G|(E)=\sup \left\{\left\|G_{z^{*}}\right\|(E):\left\|z^{*}\right\| \leq 1\right\}$.

Let $T: C(\Omega, Y) \rightarrow Z$ be a bounded linear operator. The following theorem, due to Dinculeanu and Singer, is a fortification of the Riesz representation theorem:

Theorem 7 (Dinculeanu-Singer) (Diestel and Uhl Jr 1977 p.182) There exists a unique finitely additive measure $G$ of bounded semi-variation (i.e. $|G|(\Omega)<\infty)$, defined on $\mathcal{B}_{\Omega}$ with values in $\mathcal{L}\left(Y, Z^{* *}\right)$ s.t. $T(f)=\int_{\Omega} f(\omega) d G(\omega)$ and,

(i) $G_{z^{*}}$ is a regular and countably additive Borel measure for each $z^{*} \in Z^{*}$;

(ii) the mapping $z^{*} \mapsto G_{z^{*}}$ of $Z^{*}$ into ${ }^{19} C(\Omega, Y)^{*}$ is weak ${ }^{*}$ to weak ${ }^{*}$ continuous;

(iii) $\left\langle T(f), z^{*}\right\rangle=\int_{\Omega} f(\omega) d G_{z^{*}}(\omega)$, for every $f \in C(\Omega, Y)$ and every $z^{*} \in Z^{*}$.

\footnotetext{
19 This space isomorphic to the space of regular countably additive vector measures of bounded variation on $\mathcal{B}_{\Omega}$ taking values in $Y^{*}$.
} 
Remark 7 Notice that if $T$ is positive then its representing measure $G$ is also positive. Indeed, for every $E \in \mathcal{B}_{\Omega}$ choose a sequence of continuous functions $\left(f_{n}\right)_{n=1}^{\infty} \subseteq$ $C(\Omega,[0,1])$ with $f_{n} \underset{n \rightarrow \infty}{\longrightarrow} \chi_{E}$ pointwise. Thus for every two positive elements $y \in Y$ and $z^{*} \in Z^{*}$ we have

$$
\left\langle G(E)(y), z^{*}\right\rangle=\lim _{n \rightarrow \infty} \int_{\Omega}\left(f_{n}(\omega) y\right) d G_{z^{*}}(\omega)=\lim _{n \rightarrow \infty}\left\langle T\left(f_{n} y\right), z^{*}\right\rangle \geq 0,
$$

where the first equality in Line (65) follows by combining property (i) of Theorem 7 with the bounded convergence Theorem 6 and the last inequality in that line follows from the positivity of $T$. Hence $G(E): Y \rightarrow Z^{* *}$ is a positive operator for every $E \in \mathcal{B}_{\Omega}$.

\section{Weak* operator topology}

Let $X, Y$ be Banach spaces. The operator weak ${ }^{*}$ topology on $\mathcal{L}\left(X, Y^{*}\right)$ is the weakest topology in which for every $x \in X$ the map $\mathcal{L}\left(X, Y^{*}\right) \stackrel{x}{\mapsto} Y^{*}$ given by $U \stackrel{x}{\mapsto} U(x)$ is continuous, w.r.t to the weak* topology of $Y^{*}$.

Theorem 8 The unit ball $B$ of $\mathcal{L}\left(X, Y^{*}\right)$, w.r.t. the strong topology, is compact in the operator weak* topology.

Proof The maps $\psi: \mathcal{L}\left(X, Y^{*}\right) \rightarrow \prod_{x \in X} Y^{*}$ given by

$$
\psi(U)=(U(x))_{x \in X}
$$

is continuous w.r.t. to the operator weak* topology. It is injective as $\psi(U)=\psi\left(U^{\prime}\right)$ implies $U(x)=U^{\prime}(x)$ for every $x \in X$. Thus $\psi(B)$ is a subset of

$$
\prod_{x \in X}\left\{y^{*} \in Y^{*}:\left\|y^{*}\right\| \leq\|x\|\right\}
$$

The set given in Eq. (67) is compact in the product topology, with each $Y^{*}$ endowed with its weak* topology. The set $\psi(B)$ is also closed, hence compact. Indeed, if $\psi\left(U_{\beta}\right) \underset{\beta \in B}{\longrightarrow} w$ is a converging net in $\psi(B)$ and $w \in \prod_{x \in X} Y^{*}$, then the mapping $x \stackrel{U}{\mapsto} w_{x}$ from $X$ to $Y^{*}$, is linear and is also bounded with $\|U\| \leq 1$, hence $U \in B$, and $\psi(U)=w$, so $\psi(B)$ is indeed closed. The inverse mapping from $\psi(B)$ onto $B$ is also continuous. Indeed, as $\psi$ is injective it is sufficient to prove that $\psi(V)$ is open whenever $V$ is a basic open set of $B$. By the definition of the operator weak* topology, every basic open subset of $B$ is a finite intersection of $B$ with sets of the form $\psi^{-1}\left(V^{\prime}\right)$ where $V^{\prime}$ is a basic open set of $\prod_{x \in X} Y^{*}$. But $\psi\left(B \cap \psi^{-1}\left(V^{\prime}\right)\right)=V^{\prime} \cap \psi(B)$ which is open in the topology induced on $\psi(B)$ by $\prod_{x \in X} Y^{*}$, hence $\psi^{-1}$ is continuous. Now $B=\psi^{-1}(\psi(B))$ is the continuous image of a compact set, hence compact. 


\section{References}

Aliprantis CD, Border KC (2006) Infinite dimensional analysis: a hitchhiker guide, 3rd edn. Springer, New York

Aumann RJ, Shapley LS (1974) Values of non-atomic games. Princton University Press, Princeton

Bukhvalov AV, Veksler AI, Ya G (1979) Lozanovskii, Banach lattices—some Banach aspects of their theory. Russ. Math. Surv. 34(2):159-212

Diestel J, Uhl Jr. JJ (1977) Vector measures. AMS mathematical surveys 15

Dubey P (1984) A Neyman payoffs in nonatomic economies: an axiomatic approach. Econometrica $52: 1129-1150$

Dunford N, Schwartz JT (1957) Linear operators: general theory, vol 1. Wiley, New York

Edhan O (2015) Payoffs in exact TU economies. J Econ Theory 155:152-184

Edhan O (2015) The conic property for vector measure market. Int J Game Theory 44:377-386

Haimanko O (2002) Payoffs in non-differentiable perfectly competitive TU economies. J Econ Theory 106:17-39

Hart S (1980) Measure-based values of market games. Math Oper Res 5:197-228

Kusraev AG (2000) Dominated operators, mathematics and its applications, vol 519. Springer, New York

Mertens JF (1988) The Shapley value in the non-differentiable case. Int J Game Theory 17:1-65

Mertens JF (1988) Nondifferentiable TU markets: the value. In: Roth AE (ed) The Shapley value: essays in honor of Lloyd S. Shapley, Chap. 15. Cambridge Univ. Press, Cambridge

Neyman A (2001) Values of non-atomic vector measure games. Israel J. Math. 124:1-27

Rockafellar RT (1970) Convex analysis. Princton University Press, Princeton

Shapley LS (1953) A value for n-person games, contributions to the theory of games, vol II. In: Kuhn HW and Tucker AW (eds). Princeton University Press, Princeton, pp 307-317

Williams D (1991) Probability with martingales. Cambridge University Press, Cambridge 